\title{
Evaluation of YouTube Videos as a Resource for Improving Health Literacy in Pregnant Women with Opioid Use Disorder
}

Elizabeth Kravitz, $\mathrm{MD}^{1}, \quad$ Natalie Close, $\mathrm{BSA}^{2}$

1. Baylor College of Medicine, Department of Obstetrics and Gynecology, Houston, Texas

2. University of Texas Medical Branch School of Medicine, Department of Undergraduate Medical Education, Galveston, Texas

\section{Abstract}

Background: There is significant perinatal morbidity and mortality associated with opioid use disorder.

Objective: As YouTube becomes an increasingly prevalent source for medical education among the patient population, this project aims to evaluate the utility of YouTube as a source of education on opioid use disorder (OUD).

Study Design: The first 100 YouTube videos for each of the following search terms were evaluated based on a variety of descriptive characteristics and by a scale developed from the American College of Obstetrics and Gynecology patient education resource (Table 1): "How to quit opioids during pregnancy", "opioid addiction treatment during pregnancy," and "opioid detox during pregnancy."

Results: The average views for videos targeting the general public were 20,456 views. Of these videos, the average utility score was 4.03 out of a possible 12 . There was no significant difference between the average number of views for each utility score.

Conclusion: This study not only highlights the shortcomings of current YouTube resources regarding this topic, but also emphasizes the need for further resource investment by the medical community utilizing YouTube as a resource for improving health literacy.

\section{Introduction}

The prevalence of Opioid Use Disorder (OUD) during pregnancy has paralleled the rising levels of the disease throughout the country. The significant maternal, prenatal, perinatal, and neonatal morbidity and mor- tality of this disease is well established. Babies born from mothers using opioids have an increased risk of fetal growth restriction, placental abruption, fetal death, preterm labor, and intrauterine passage of meconium. ${ }^{1}$ Withdrawal during pregnancy similarly holds a risk of preterm birth, fetal stress, and even fetal death. The increasing prevalence of associated 
risks is exacerbated by poor health literacy, as recent evidence has demonstrated an association between low health literacy and higher frequencies of preterm birth and small for gestational age babies. ${ }^{2}$ In order to address and minimize these risks, patient education is crucial. Around the world people of all backgrounds, education levels, and incomes are looking to YouTube as a source of medical education. ${ }^{3}$ The purpose of our project was to evaluate the utility of YouTube videos as a source of medical education on opioid use disorder during pregnancy.

\section{Materials \& Methods}

A YouTube search was conducted on October 26th, 2019 with the following search terms: "How to quit opioids during pregnancy," "opioid addiction treatment during pregnancy," and "opioid detox during pregnancy." The first 100 videos for each search term, sorted by relevance, were saved and videos were excluded if they were duplicates, silent videos, in a language other than English, or if there was no mention of opioid use disorder or pregnancy. They were analyzed on the following descriptive characteristics: length, number of views, date published, targeted audience (professionals or patients/general public), and video source type (personal, professional, news, webinar/conference). A 12-point scale was developed matching the American College of Obstetrics and Gynecology patient education resource (Table 1). This scale was applied to each video in order to evaluate its utility for a patient population. Videos were sorted based on how many of the 12 points were included. Less than 3 points were deemed poor utility, 3.0 to 5.9, mild utility, 6.0 to 8.9 , moderate utility, and 9.0 to 12 , excellent utility. Due to the qualitative nature of the key areas of content utilized in the scale, if there was not a clear discrimination on if the content was present or not, the authors deliberated between themselves. The primary outcome was to assess the use and utility of these videos by the general public by average views and by the aforementioned scoring criteria. Secondary outcomes included specific observations on the presence or absence of each key area of content in these videos. Statistical evaluation was performed with the Student's T-test. This was a descriptive study and it did not require IRB approval as only a public resource was reviewed.

\begin{tabular}{|c|c|}
\hline Key Areas of Content & Points \\
\hline What are opiods? & 1 \\
\hline What defines opiod substance abuse? & 1 \\
\hline Prescription opiods can lead to abuse. & 1 \\
\hline \multicolumn{2}{|l|}{ What are the risks during a pregnancy? } \\
\hline Placental abruption & 0.5 \\
\hline Prenatal complications & 0.5 \\
\hline Preterm birth / labor & 0.5 \\
\hline Stillbirth & 0.5 \\
\hline Neonatal Abstinence Syndrome & 1 \\
\hline \multicolumn{2}{|l|}{ How is it treated during pregnancy? } \\
\hline Methadone & 0.5 \\
\hline Buprenorphine & 0.5 \\
\hline Explanation of access / administration of medication & 1 \\
\hline Benefits of treatment & 1 \\
\hline Risks of treatment & 1 \\
\hline Breastfeeding on Methadone / Buprenorphine & 1 \\
\hline How to get help & 1 \\
\hline
\end{tabular}

\section{Results}

Of the 300 videos in total, 113 remained after exclusion criteria was applied, 86 of those had a targeted audience of a patient or the general public. The source type of the videos varied from professional videos (36), personal videos (17), news clips (31), and webinars/conferences (2). The primary outcome, the average views for videos targeting the general public, was 20,456 views. Of these videos, the average utility score was 4.03. Three of these videos qualified as excellent utility, 19 were moderate utility, 35 were mild 
utility, and 29 were poor utility. There was no significant difference between the average number of views for each utility score ( $p$-value $>0.05$ ), which can be seen in Table 2.

\begin{tabular}{|l|c|}
\hline Utility Score (N) & Mean Number of Views* \\
\hline $\begin{array}{l}\text { Excellent (3) } \\
9.0-12.0\end{array}$ & $141514.67^{*}$ \\
\hline $\begin{array}{l}\text { Moderate (19) } \\
6.0-8.9\end{array}$ & $18909.21^{*}$ \\
\hline $\begin{array}{l}\text { Mild (35) } \\
3.0-5.9\end{array}$ & $133275.80^{*}$ \\
\hline $\begin{array}{l}\text { Poor (29) } \\
0<3.0\end{array}$ & $382330.97^{*}$ \\
\hline
\end{tabular}

*p-value not significant (p-value $>0.05$ )

(Table 2)

Comparison of mean number of views for videos by utility score

In a comparison of videos created by healthcare professionals (e.g., professional videos and webinars/ conferences) versus the non-professionally made videos (e.g., personal videos and news clips), the average utility scores were 4.28 and 3.83 , respectively (Table

\begin{tabular}{|l|c|c|c|}
\hline & $\begin{array}{c}\text { Source: Healthcare } \\
\text { Professionals } \\
\text { N=38 }\end{array}$ & $\begin{array}{c}\text { Source: } \\
\text { Non-Healthcare } \\
\text { Professionals } \\
\text { N=48 }\end{array}$ & P-Value \\
\hline $\begin{array}{l}\text { Mean } \\
\text { Utility } \\
\text { Score }\end{array}$ & 4.28 & 3.83 & $0.419^{*}$ \\
\hline $\begin{array}{l}\text { Mean } \\
\text { Length } \\
\text { Minutes }\end{array}$ & 9 & 4.4 & 0.034 \\
\hline $\begin{array}{l}\text { Mean } \\
\text { Number } \\
\text { Views }\end{array}$ & $68,583.34$ & $302,596.04$ & $0.257^{*}$ \\
\hline
\end{tabular}

+ "profressional videos" \& webinars

++ "personal videos" \& news clips

* Notes: *p-value not significant ( $p$-value $>0.05$ )

(Table 3)

Comparison of scores of videos created by healthcare professionals vs. by non-healthcare professionals
3). The difference between these values was found to be statistically not significant $(\mathrm{p}$-value $=0.419)$. The number of views on healthcare-created videos was found to be less, on average, than non-healthcare-created videos (68,583 vs. 302,596 views, respectively), though this was not statistically significant as well ( $\mathrm{p}$-value $=0.257)$. There was a statistically significant difference in the mean length of videos created by healthcare professionals versus the non-healthcare created videos (9 minutes vs. 4.4 minutes, respectively; p-value $=0.034)$.

Our secondary outcomes yielded the results seen in Table 4. Notably, one half failed to define opioid use disorder, and $48 \%$ failed to define what an opioid is (Table 4).

\begin{tabular}{|c|c|c|}
\hline Characteristic of Video & Result & $\begin{array}{c}\text { Percentage } \\
\mathrm{N}=\mathbf{8 6}\end{array}$ \\
\hline Views (average) & 20,456 views & \\
\hline Average score & 4.03 & \\
\hline Defines opioids & 45 videos & $52 \%$ \\
\hline Defines opioid use disorder & 43 videos & $50 \%$ \\
\hline $\begin{array}{l}\text { Includes consequences of } \\
\text { OUD during prenatal period }\end{array}$ & 23 videos & $27 \%$ \\
\hline $\begin{array}{l}\text { Neonatal abstinence } \\
\text { syndrome }\end{array}$ & 61 videos & $71 \%$ \\
\hline Ability to breastfeed & 2 videos & $2 \%$ \\
\hline Mentions treatment & 55 videos & $64 \%$ \\
\hline Includes how to get help & 22 videos & $26 \%$ \\
\hline \multicolumn{3}{|l|}{ Source type: } \\
\hline Professional & 36 videos & $42 \%$ \\
\hline Personal & 17 videos & $20 \%$ \\
\hline News & 31 videos & $36 \%$ \\
\hline Webinar & 2 videos & $2 \%$ \\
\hline
\end{tabular}

(Table 4)

Data on videos with targeted audience of patient (total 86 videos) 


\section{Discussion}

\section{Principal Findings:}

YouTube videos are a highly utilized resource for education by pregnant mothers searching for help with OUD, however, these videos are lacking in breadth and quality of medical knowledge.

\section{Results:}

Recognition and treatment of opioid use disorder during pregnancy allows for prevention of the direct risks of opioids to mother and fetus, in addition to indirect risks such as transmission of HIV and hepatitis $B$ through the sharing of needles. The availability of treatment may be the most influential information to be included in patient education, as it offers hope and encouragement to mothers. Yet, only $26 \%$ of videos offered information directing mothers to help.

Medication assisted treatment (MAT) is an evidence-based practice utilizing medications such as buprenorphine or methadone to alleviate cravings and withdrawal. ${ }^{4}$ This practice was only mentioned in $55(64 \%)$ of the videos, even though MAT is proven to be superior to other forms of treatment for relapse prevention. ${ }^{4}$ We found that these resources failed to promote the mother-baby dyad, with a particular focus $(71 \%)$ on Neonatal Abstinence Syndrome, but little acknowledgment of the maternal risks (27\%).

Some videos offered anecdotes commenting on the benefits of MAT therapy, others provided anecdotes of self-medicating with Kratom, an opioid agonist purchased easily and legally on the internet. This poses the obvious risk of dosing, as women not under supervision of a healthcare provider, can overdose while attempting to self-manage opioid withdrawal. ${ }^{4}$ Additionally, pregnant women attempting to self-manage withdrawal can put the fetus at risk, with a risk of fetal distress and even fetal demise.

Unexpectedly, the average utility score of the videos produced by healthcare professionals was not significantly higher than the videos produced by non-healthcare professionals (4.28 vs. 3.83 , respectively, with $\mathrm{p}$-value $=0.419$ ). This suggests that the videos currently available on YouTube are largely lacking in comprehensive, patient-oriented information regard ing OUD during pregnancy, regardless of source. This highlights a need from the medical community to increase not only the quantity, but the quality of medical education resources for pregnant patients with OUD on accessible platforms such as YouTube. Additionally, videos may be more effective and garner more views if they are kept to a shorter length (e.g., 5 minutes or less), as shorter videos tended to have more views on average.

\section{Clinical Implications:}

The findings presented suggest a need by the medical community to invest in the development of comprehensive medical education for patients with OUD, to ensure that patients seeking education online are not introduced to misleading, or incorrect, data.

\section{Research Implications:}

Further research should be done to evaluate patient perception of these videos and survey the outcomes of women receiving medical education from online resources such as education found on social media sites such as YouTube.

\section{Strengths and Limitations:}

A strength of the study was the large sample size of videos reviewed, given that the majority of YouTube users watch within the first 30 videos, ${ }^{5}$ much less than the videos evaluated by the authors. However, YouTube algorithm currently individualizes the presentation of videos, therefore, sorting can vary. ${ }^{5}$ Given that the largest components in this sorting algorithm is relevance, engagement, and quality, the authors felt that most videos evaluated were those that would be viewed by the general patient audience. ${ }^{6}$

A weakness of the study was that neither authors were experts in the field of substance use disorder or neonatology (both authors were medical students at the time of review). The videos were divided into two groups, with each author watching their own portion of the YouTube videos, posing the risk of discrepancy in scoring between the two groups.

\section{Conclusion}


YouTube videos offer a platform for health education that can address people of varying education levels regardless of the geographic location-whether they live in a medically underserved area or live in a community with a high density of medical providers. These findings highlight a need for a shift in patient education towards YouTube and other accessible platforms.

\section{References}

1. Committee opinion No. 711: Opioid use and opioid use disorder in pregnancy. Obstetrics \& Gynecology. 2017;130(2):e81-e94. doi:10.1097/ aog.0000000000002235

2. Yee LM, Silver RM, Haas DM, et al. 8: Association between health literacy and maternal and neonatal outcomes. American Journal of Obstetrics and Gynecology. 2020;222(1):S6-S7. doi:10.1016/j. ajog.2019.11.024

3. Tackett S, Slinn K, Marshall T, Gaglani S, Waldman V, Desai R. Medical Education Videos for the World: An Analysis of Viewing Patterns for a YouTube Channel. Academic Medicine. 2018;93(8):11501156. doi: $10.1097 / \mathrm{acm} .0000000000002118$

4. Chavan N, Ashford KB, Wiggins A, Critchfield A. 729: Buprenorphine for medication assisted treatment (MAT) of opioid use disorders in pregnancy: Relationship to neonatal opioid withdrawal syndrome (NOWS). American Journal of Obstetrics and Gynecology. 2017;216(1):S425-S425. doi:10.1016/j. ajog.2016.11.463

5. Özdal Zincir Ö, Bozkurt AP, Gaş S. Potential patient education of youtube videos related to wisdom tooth surgical removal. Journal of Craniofacial Surgery. 2019;30(5):e481-e484. doi:10.1097/ scs.0000000000005573

6. Youtube. (n.d.). Product Features: YouTube search. Retrieved December 28, 2020, from https:// www.youtube.com/howyoutubeworks/product-features/search/ 